\title{
Maximizing Trip Coverage in the Location of a Single Rapid Transit Alignment
}

GILBERT LAPORTE gilbert@crt.umontreal.ca Canada Research Chair in Distribution Management, HEC Montréal, 3000 Chemin de la Côte-Sainte-Catherine, Montréal, Canada H3T 2A7

JUAN A. MESA

Departamento de Matemática Aplicada II, Escuela Técnica Superior de Ingenieros Industriales,

Universidad de Sevilla, Camino de los Descubrimientos s/n, 41092 Sevilla, Spain

FRANCISCO A. ORTEGA

riejos@us.es

Departamento de Matemática Aplicada I, Escuela Técnica Superior de Arquitectura, Universidad de Sevilla, Avda. Reina Mercedes s/n, 41012 Sevilla, Spain

IGNACIO SEVILLANO

sevillano@terra.es

Production Management, Rótulos Mediterráneo, Polígono Industrial Las Chaparrillas, Parcela 1, Nave 5-6, 41016 Sevilla, Spain

\begin{abstract}
This article describes several heuristics for the construction of a rapid transit alignment. The objective is the maximization of the total origin-destination demand covered by the alignment. Computational results show that the best results are provided by a simple greedy extension heuristic. This conclusion is confirmed on the Sevilla data for scenarios when the upper bound for inter-station distance is greater than $1250 \mathrm{~m}$. Otherwise, when those upper bounds are smaller $(750 \mathrm{~m}$ and $1000 \mathrm{~m})$, an insertion heuristic followed by a post-optimization phase yields the best results. Computational times are always insignificant.
\end{abstract}

Keywords: transit systems, trip coverage, effectiveness, heuristics

\section{Introduction}

The construction of rapid transit systems such as metro networks and light railways is gaining in popularity in large cities faced with traffic problems. A number of major decisions must be made when designing such systems. The planning process is usually long and involves several players such as civil and traffic engineers, city planners, politicians, environmentalists, citizen interest groups, etc. The rapid transit design problem contains two intertwined location problems: determining an alignment and locating stations on it. Two approaches are used for this problem. The first initially locates the main stations and then implicitly determines the alignment by locating the remaining stations between those that were fixed in the first step. This was the approach used for the extension of the Lisbon metro (Viegas and Mexia, 1993). In the second approach the alignment is first determined and stations are only located afterwards. This is the approach used for 
the design of the Sevilla metro now under construction. The final design is actually a refinement of a preliminary proposal, taking into account population density, employment zones, existing bus lines, and the main transportation corridors within the city (Junta de Andalucía, 2002). In both approaches the forecasted traffic level can be determined by means of a four-step planning model: trip attraction and generation, trip distribution, mode choice, and assignment.

Numerous criteria are used for the assessment of potential solutions and it has long been recognised (see, e.g., Magnanti and Wong, 1984) that while classical operational research models can help streamline the evaluation and decision process, they cannot by themselves solve the problem in its entirety. A sensible approach for tackling difficult design problems is to provide decision makers with a rich set of quality solutions that can then be assessed and presented to decision makers (Laporte, 1995). In recent years several operational research tools have been proposed for the design of rapid transit networks (see the recent survey article by Laporte, Mesa, and Ortega, 2000). The present study extends some of the existing work.

The primary goal when designing a rapid transit system is to provide shortest travel times in order to improve the population's mobility (Gendreau, Laporte, and Mesa, 1995). Since the expected number of trips is assumed to be related to the population covered by the system (people who live or work around the lines), one of criteria most frequently used is the maximization of coverage provided by the lines.

Basically, three approaches have been proposed to measure the coverage of an alignment. The first, used by Chapleau, Lavigueur, and Baass (1986) and Wirasinghe and Vandebona (1987) consists of drawing embedded corridors around the alignment and of assigning coverage factors to each corridor. Coverage gradually decreases as the walking distance to the alignment decreases and eventually falls to zero (figure 1). The corridor approach is rather unsatisfactory since it ignores the fact that someone living close to a metro line but far from a station will not be attracted by the line. This is why other methods are often used to measure coverage.

A second approach consists of determining catchment areas around each station of the alignment. As in the previous method, concentric geometrical shapes with decreasing

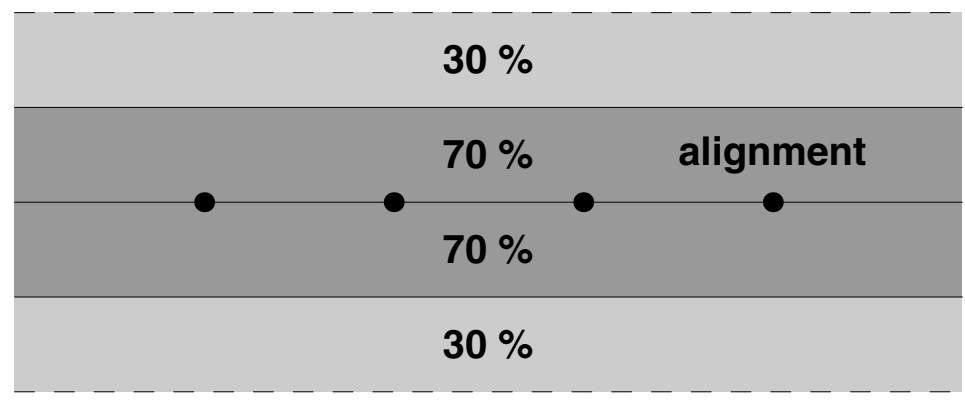

Figure 1. Alignment with two embedded corridors around it. The values show the percentage of population living in a given corridor likely to use the transit system. 


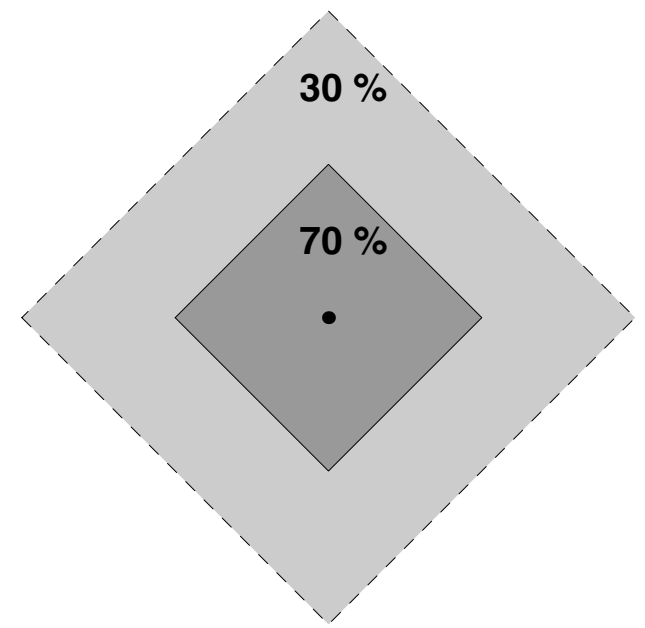

Figure 2. Station with embedded catchment zones around it.

attraction factors can be built around each station (figure 2). This is essentially the approach taken by Dufourd, Gendreau, and Laporte (1996) and by Bruno, Gendreau, and Laporte (2002) who have discretized the demand around each station.

For each access mode (walking, park-and-ride, kiss-and-ride, feeder bus, etc.) a representative planar metric $d(\cdot, \cdot)$ is used to estimate time spent travelling from origin point $X$ in the city to destination point $s$ (station). Walking distances are typically measured by means of the Manhattan metric (Larson and Odoni, 1981; Lutin and Benz, 1992; Dufourd, Gendreau, and Laporte, 1996; Bruno, Gendreau, and Laporte, 2002) when the predominant street pattern is a rectangular grid, but other approximations are possible such as block norms (Ward and Wendell, 1980), the $\tau$-inflated $l_{p}$ norm (Love, Morris, and Wesolowsky, 1988), and combinations of the Manhattan and Euclidean norms (Brimberg and Love, 1993).

Once a metric $d$ has been fixed, the isochronic curve for walking time $r>0$, centred at station point $s$, generates a catchment zone

$$
B(s, r)=\left\{x \in \mathbb{R}^{2}: d(x, s) \leq r\right\}
$$

in which the number of potential users for the transit system must be estimated by collecting population data provided by the census tracts overlapping $B(s, r)$. Since the shape of census tracts is polygonal and sometimes non-convex, a triangulation must first be carried out to reduce the number of cases to be analysed when evaluating the intersection between $B(s, r)$ and the census tract (Laporte, Mesa, and Ortega, 2002).

One major drawback of the catchment model is that it ignores station-to-station ridership demand. Thus a person travelling along the North-South axis is unlikely to be attracted to a station located on an East-West alignment (assuming of course this alignment is not part of a larger interconnected network). This is why a third approach, based 
on actual demand between origin/destination $(\mathrm{O} / \mathrm{D})$ pairs, seems more appropriate. The data required for this model (O/D information, population distribution in each census tract and geometrical shape of census tracts) are more voluminous but usually available from government agencies; moreover, these data can be visualised by using a Geographical Information System (GIS) and integrated with an optimization software. Although this third approach requires a more substantial data gathering and computational effort, it is based on more realistic assumptions.

Our purpose is to present an alignment location methodology based on O/D information, therefore including the three first steps of the four-stage classical transit planning methodology. It contains two main parts. In Section 2 we describe a model for the estimation of the actual demand between two stations. In Section 3 we propose simple heuristics for the design of an alignment maximizing trip coverage. The heuristics can be applied to any O/D demand matrix, i.e., they can work independently of the model used to estimate O/D demand. Computational results are presented in Section 4, followed by conclusions in Section 5.

\section{Modeling origin/destination demand}

In this section we extend the station catchment area model of Laporte, Mesa, and Ortega (2002) to a more involved trip coverage model.

\subsection{Population covered by a station}

Planners often use data provided by city census tracts to evaluate the population covered by a station. In general, each census track is a non-convex polygon in which users are attracted to a station according to a gravity model (Ortúzar and Willumsen, 1990).

Let $C=\left\{c_{h}: h=1, \ldots, H\right\}$ be the set of census tracts and $\rho_{h}$ the respective density of each $c_{h} ; h=1, \ldots, H$. Then the coverage $R(i)$ provided by station $s_{i}$ is defined by

$$
R(i)=\sum_{h=1}^{H} \sum_{k=1}^{K} \frac{a \rho_{h}}{{\overline{r_{k}}}^{2}} \operatorname{Area}\left(\left(B_{k} \backslash B_{(k-1)}\right) \cap c_{h}\right) ; \quad i=1, \ldots, n,
$$

where $k$ is a ring index (figure 3), $K$ is the number of rings per station, $B_{k} \backslash B_{(k-1)} \equiv$ $B\left(s_{i}, r_{k}\right) \backslash B\left(s_{i}, r_{k-1}\right)$ is the ring centered at $s_{i}$ and comprised between radii $r_{k-1}$ and $r_{k}$, $\overline{r_{k}}$ is an intermediate value between radii $r_{k}$ and $r_{k-1}$ (for instance, the discrete average $\left(r_{k}+r_{k-1}\right) / 2$ or another continuous average where directional bias for weighted $l_{p}$ norms is taken into account, as pointed in Brimberg, Love, and Walker (1995)), and $a$ is a parameter to be calibrated. 


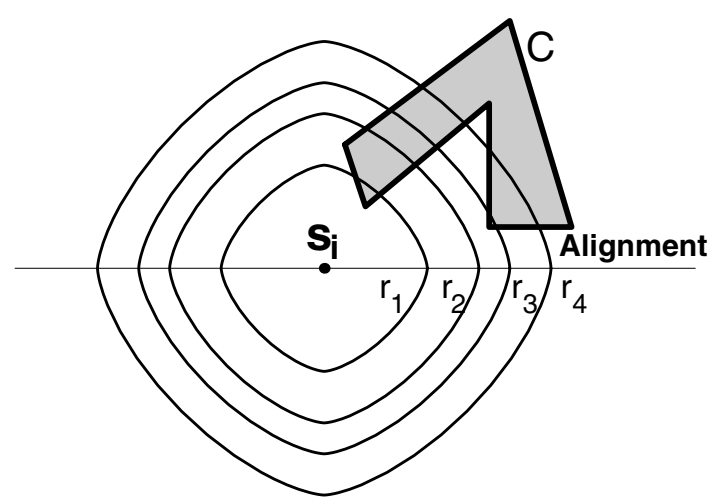

Figure 3. Coverage from station $s_{i}$.

\subsection{Trip coverage}

To combine the estimation of passenger O/D patterns with the notion of population coverage, Mesa and Ortega (2001) have proposed the following approach which is illustrated by figure 4 .

Each station pair $(i, j)$ has an associated $K \times K$ matrix, denoted by $O D_{i j}$, whose elements $O D_{i j}\left(k, k^{\prime}\right.$ ) (for all $k, k^{\prime}=1, \ldots, K$ ) represent the weighted sum of portions of values $t_{l m}$ (predicted number of trips produced in zone $z_{l}$ and attracted to zone $z_{m}$, for all $l, m=1, \ldots, L)$. Weights are defined by taking into consideration attraction radii $r_{i}$ and $r_{i^{\prime}}$ (respectively, at origin and destination stations), yielding the following O/D values:

$O D_{i j}\left(k, k^{\prime}\right)=\sum_{l, m=1,(l \neq m)}^{L} \frac{a^{2}}{{\overline{\bar{r}_{k}}}^{2}{\overline{r^{\prime}}}^{2}} \frac{\operatorname{Area}\left(\left(B_{i k} \backslash B_{i(k-1)}\right) \cap z_{l}\right)}{\operatorname{Area}\left(z_{l}\right)} \frac{\operatorname{Area}\left(\left(B_{j k^{\prime}} \backslash B_{j\left(k^{\prime}-1\right)}\right) \cap z_{m}\right)}{\operatorname{Area}\left(z_{m}\right)} t_{l m}$.

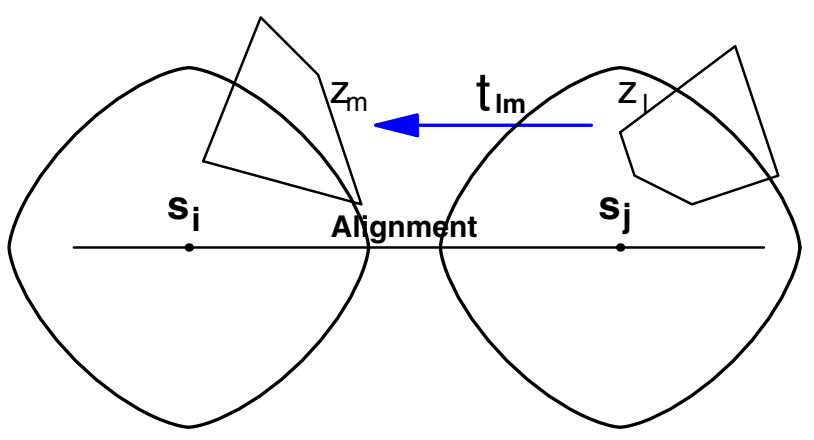

Figure 4. Passenger flow between a pair of stations. 
Therefore, the trip coverage $f_{i j}$ provided by a pair of different stations $\left(s_{i}, s_{j}\right)$ can be obtained by adding all matrix elements:

$$
f_{i j}=\sum_{k, k^{\prime}} O D_{i j}\left(k, k^{\prime}\right) .
$$

\subsection{Network effectiveness}

The next step in the modelling approach is to scale the $f_{i j}$ values to obtain a so-called effectiveness measure reflecting the user benefit derived from using the transit system. Several mode choice models are available (see, e.g., the recent survey by BouzaïeneAyari, Gendreau, and Nguyen, 2001). In this study we have opted for a simple logit model to desegregate the demand.

The model assumes that potential users choose between only two transport modes:

- a transit line $\operatorname{Align}(E)$, defined on a set $E$ of stations;

- a private car that uses the street grid.

In what follows, $\tau_{i j}(E)$ denotes the time of the quickest path connecting nodes $s_{i}$ and $s_{j}$ using the network based on $E$, and $\tau_{i j}^{A}$ represents the time of the quickest path connecting nodes $s_{i}$ and $s_{j}$ by using another means of transport. The proportion of trips between stations $s_{i}, s_{j}$, using the rapid transit network on set $E$, is obtained by means of the formula

$$
g_{i j}(E)=\frac{1}{1+e^{-\gamma\left(\tau_{i j}^{A}-\tau_{i j}(E)\right)}},
$$

where $\gamma$ is a positive parameter to be calibrated.

The effectiveness measure we use is obtained by summing up the terms $n_{i j}(E)=$ $f_{i j} g_{i j}(E)$ over $i$ and $j$, where $f_{i j}$ denotes trip coverage between stations $s_{i}$ and $s_{j}$, and $g_{i j}(E)$ indicates the value of the logit function (see figure 5) associated with origindestination pair $\left(s_{i}, s_{j}\right)$ when an alignment on the nodes of set $E$ is considered. Once the parameters are adequately calibrated, each effectiveness value $n_{i j}(E)$ can be used to represent the passenger flow between stations $s_{i}$ and $s_{j}$ in the presence of an alignment on $E$.

\section{Formulation and heuristics}

We are interested here in locating an alignment through a set $E$ of stations in order to maximize total traffic flow, subject to a length constraint. Formally the problem can be stated as follows. 
User-Profit (probability)

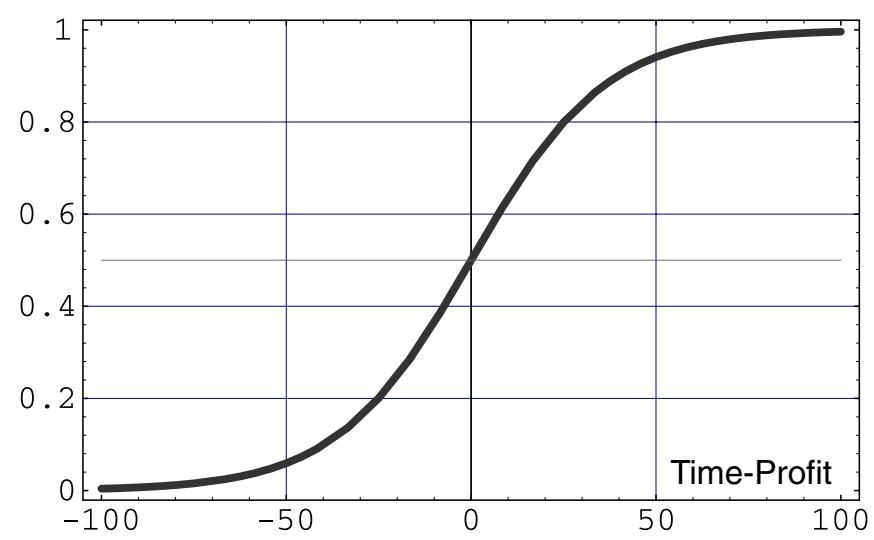

Figure 5. Logit function.

Given a set of candidate nodes $S=\left\{s_{1}, s_{2}, \ldots, s_{n}\right\}$, determine a subset $E$ of $S$ and a non-intersecting alignment Align $(E)$ on points of $E$ yielding a maximal ridership, subject to a maximum length constraint LMAX.

In other words,

$$
(M T C P): \begin{cases}\underset{E \subset S}{\operatorname{maximize}} & \sum_{i, j} n_{\left(i \neq j ; s_{i}, s_{j} \in E\right)}(E) \\ \text { subject to } & \operatorname{Length}[\operatorname{Align}(E)] \leq L M A X\end{cases}
$$

Since the problem of determining a bounded length Hamiltonian path in a graph is $N P$-hard (Garey and Johnson, 1979), the maximal trip coverage problem $(M T C P)$ is also $N P$-hard. Hence, it is important to develop heuristics to solve (MTCP) effectively. We have therefore devised two constructive heuristics and a post-optimization procedure which are outlined below. Simultaneously, the behaviour of some of the proposed heuristics was assessed in a real setting.

Sevilla is the capital city of the autonomous Andalucía region with more than one million inhabitants in the metropolitan area. It is one of the 22 European cities currently planning a metro network (see www.metropla.net). Only Line 1 (19 km, 23 stations) is currently being constructed in the East-West corridor crossing the city centre. A set of 21 candidate nodes were randomly chosen in Sevilla in order to illustrate the behaviour of the proposed heuristics for $L M A X=19 \mathrm{~km}$. In this simplified scenario, nodes numbered $3,11,12$ and 15 are interior to the central circle which represents the historic-commercial centre of Sevilla (see figure 6).

The central circle is the most attractive destination of trips originating in other residential areas, as can be observed in figure 7 where the thickness of each edge is 


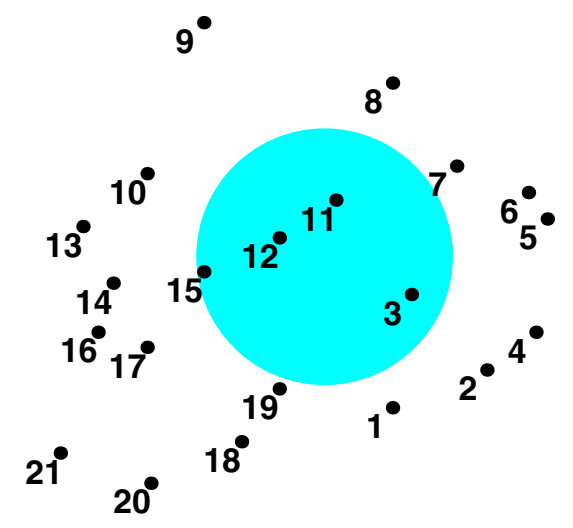

Figure 6. Nodes of the Sevilla example.

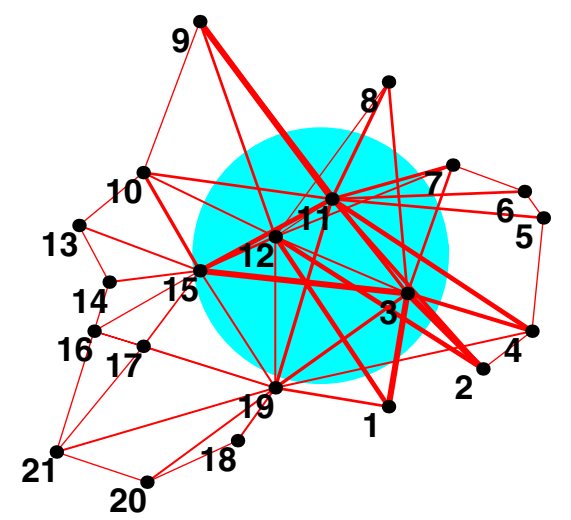

Figure 7. Flow between nodes in the Sevilla example.

proportional to the attraction level between nodes (for clarity, the low demand edges are not shown).

\section{H1: Greedy extension of an alignment}

This approach may be viewed as a greedy algorithm since the best possible decision is made at each stage without regard to later decisions.

\section{Constructive heuristic $\mathrm{H} 1$}

1. Include in $\operatorname{Align}(E)$ the edge $\left(s_{i}, s_{j}\right)$ (i.e., $\left.E=\left\{s_{i}, s_{j}\right\}\right)$ yielding $\max \left\{n_{i j}\right\}$.

2. Extend the current alignment $\operatorname{Align}(E)$ at either end by adding a new edge, $\left(s_{k}, s_{i}\right)$ or $\left(s_{j}, s_{k}\right)$, yielding the maximum objective $\sum_{\forall s_{i}, s_{i} \in E} n_{i j}(E)$, not intersecting the current alignment, and not causing Length[Align $(E)]$ to exceed $L M A X$. Repeat until the current alignment can no longer be extended. 


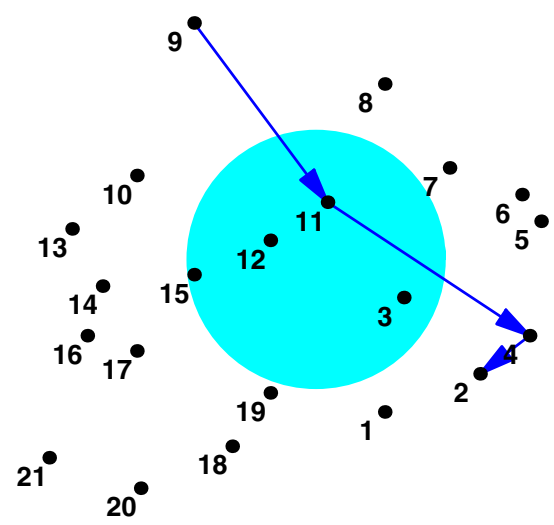

Figure 8. Alignment after greedy extensions.

Figure 8 shows the final alignment (nodes 9-11-4-2) maximizing trip coverage obtained by applying the heuristic $\mathrm{H} 1$.

\section{H2: Extension of an alignment through greedy insertions}

The underlying idea of these improvement heuristics is to start with a feasible solution and, while maintaining feasibility, to extend the current set $E$ of selected nodes by successive insertions. Again, decisions are made at each stage in a greedy fashion.

\section{Constructive heuristic $\mathrm{H} 2$}

1. Include in $\operatorname{Align}(E)$ the edge $\left(s_{i}, s_{j}\right)$ yielding $\max \left\{n_{i j}\right\}$.

2. Consider in turn for each non-inserted station and insert it in the current alignment according to one of the following three variants:

\section{Variant A}

- Determine for each non-inserted station the insertion position yielding the least increase in $\operatorname{Length}[\operatorname{Align}(E)]$ and compute the added ridership that would result from this best insertion.

- Extend Align $(E)$ by inserting the non-inserted station (in its best position) for which the added ridership is maximized.

\section{Variant B}

- Determine for each non-inserted station the insertion position yielding the least increase in $\operatorname{Length}[\operatorname{Align}(E)]$ (the best insertion).

- Extend $\operatorname{Align}(E)$ by inserting the non-inserted station (in its best position) for which the added length is minimized. 
- Break ties by using the maximal added ridership criterion.

\section{Variant C}

- Determine for each non-inserted station the insertion position yielding the least increase in Length[Align $(E)]$ and compute the added ridership that would result from this best insertion.

- Extend Align $(E)$ by inserting the non-inserted station (in its best position) for which the ratio $\frac{\text { (increased ridership) }}{\text { (increased length) }}$ is maximized.

For the same example, figure 9 describes the final alignment (nodes 9-11-3-2-4) obtained with $\mathrm{H} 2(\mathrm{~A})$, whereas the corresponding best network after using variant $\mathrm{B}$ of heuristic H2 (nodes 9-11-12-15-14-16) is shown in figure 10.

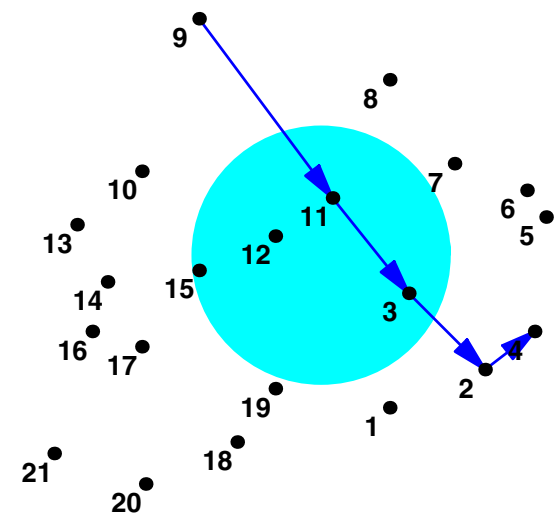

Figure 9. Alignment after greedy insertions (A).

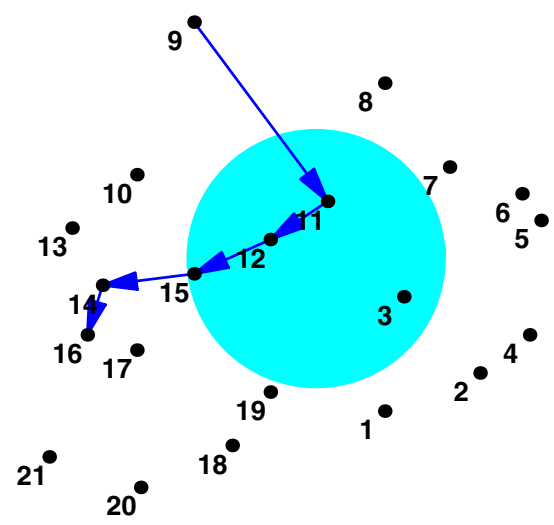

Figure 10. Alignment after greedy insertions (B). 


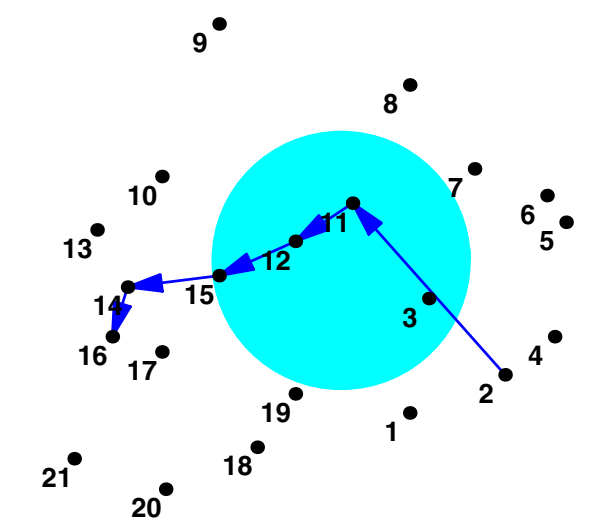

Figure 11. Alignment after greedy insertions (C).

The alignment shown in figure 11 corresponds to the execution of algorithm $H 2(C)$, where the initial tie between edges $(9,11)$ and $(2,11)$ was broken in favor of $(2,11)$ because of its larger ridership/length ratio.

\subsection{Post-optimization procedure for $\mathrm{H} 2$}

The objective value of the solution produced by heuristic $H 2$ depends on the order of the nodes in the alignment, which is gradually constructed. Therefore, a post-optimization procedure acting on the node sequence may improve the objective.

\section{Post-optimization procedure for $\mathrm{H} 2$}

1. Given a solution produced by $H 2$, remove in turn each station, link its predecessor to its sucessor, and reinsert the station just removed using the same variant as in the construction phase.

2. Stop when no station removal and reinsertion can yield an improvement in the objective.

The post-optimization procedure was applied to the three earlier alignments, obtained after using $H 2(A, B, C)$ in order to improve global effectiveness but never produced any improvement.

\section{Computational results}

\subsection{Test on simulated context}

The heuristics just described were coded in Pascal and run on a Sunfire 4800 Computer $(900 \mathrm{MHz})$ and tested on randomly generated instances. We constructed artificial cities 

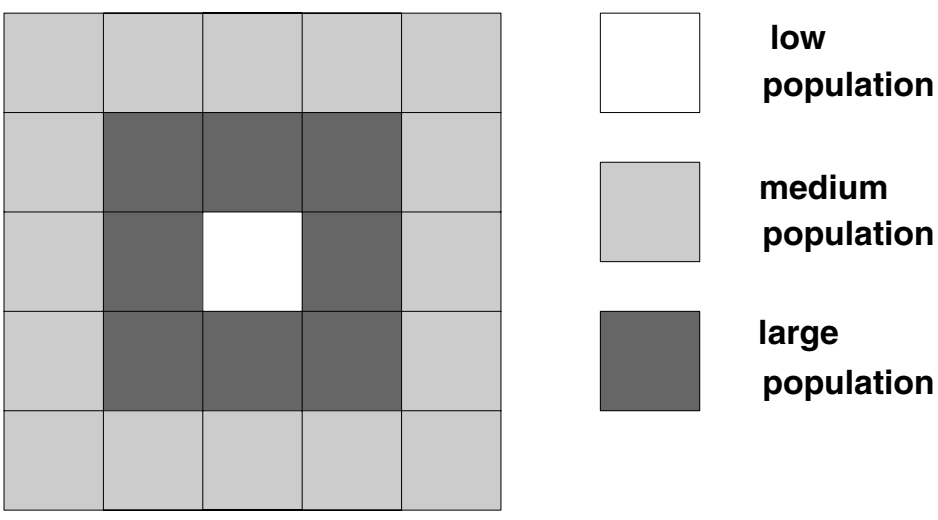

medium

population

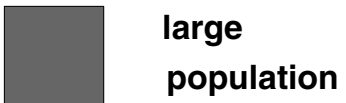

Figure 12. Simulated city subdivided into 25 equal zones.

\begin{tabular}{|c|c|c|c|}
\hline to & $\begin{array}{c}\text { Central } \\
\text { zone }\end{array}$ & $\begin{array}{c}\text { Intermediate } \\
\text { zone }\end{array}$ & $\begin{array}{c}\text { Suburban } \\
\text { zone }\end{array}$ \\
\hline $\begin{array}{c}\text { Central } \\
\text { zone }\end{array}$ & 0.60 & 0.04 & 0.005 \\
\hline $\begin{array}{c}\text { Intermediate } \\
\text { zone }\end{array}$ & 0.36 & 0.06 & 0.01 \\
\hline $\begin{array}{c}\text { Suburban } \\
\text { zone }\end{array}$ & 0.20 & 0.06 & 0.02 \\
\hline
\end{tabular}

Figure 13. Travel probabilities for the random instances.

on the $[0,21]^{2}$ square. The square was subdivided into 25 equal zones where the central zone, corresponding to the city center, has a low population, the eight intermediate zones surrounding it have a large population and the remaining suburban zones have a medium population (figure 12).

More specifically we randomly generated populations in the interval [2000, 5000] for the central zone, in [15000, 20000] for the intermediate zones and in $[5000,10000]$ for the suburban zones. We then defined travel probabilities $p_{k l}$ between zones of each type as in figure 13. These values mean that any resident from a zone of type $k$ has a probability $p_{k l}$ of traveling to a zone of type $l$. Note that the probabilities of each line add up to 1 if the second column is multiplied by 8 and the third column is multiplied by 16 . We superimposed the lattice of integer coordinate points in the $[0,21]^{2}$ square onto the city, which yielded 484 potential locations for stations. The population of each zone was then equally divided between the points of the zone.

We then constructed a bounded length single alignment over the artificial city with $L M A X=10,20$ and 40. Preliminary results produced compact and zigzaging alignments tending to remain within a single zone. To remedy this we introduced a linearity coefficient $\lambda$ to force the alignment to have a more linear shape. This coefficient is the length of the alignment divided by the length of the straight line between its two extremities. In other 
Table 1

Summary of computational results

\begin{tabular}{|c|c|c|c|c|c|}
\hline Heuristic & LMAX & Length & $\begin{array}{c}\text { Ridership: } \\
\text { number of passengers } \\
\text { using the alignment }\end{array}$ & $\begin{array}{l}\text { Ridership to } \\
\text { length ratio }\end{array}$ & Seconds \\
\hline \multirow[t]{3}{*}{ H1 } & 10 & 9.6 & 114.1 & 11.9 & 0.00 \\
\hline & 20 & 19.4 & 388.7 & 20.0 & 0.02 \\
\hline & 40 & 37.7 & 661.3 & 17.6 & 0.03 \\
\hline \multirow{3}{*}{$\mathrm{H} 2$ (A) } & 10 & 7.9 & 50.4 & 6.2 & 0.02 \\
\hline & 20 & 11.4 & 69.1 & 6.1 & 0.02 \\
\hline & 40 & 26.2 & 88.3 & 3.4 & 0.02 \\
\hline \multirow{3}{*}{$\begin{array}{l}\mathrm{H} 2 \text { (A) } \\
\quad+\text { post- } \\
\quad \text { optimization }\end{array}$} & 10 & 7.9 & 50.4 & 6.4 & 0.02 \\
\hline & 20 & 11.4 & 69.1 & 6.1 & 0.02 \\
\hline & 40 & 26.2 & 88.3 & 3.4 & 0.02 \\
\hline \multirow[t]{3}{*}{ H2 (B) } & 10 & 8.0 & 44.9 & 5.6 & 0.02 \\
\hline & 20 & 12.6 & 68.3 & 5.4 & 0.02 \\
\hline & 40 & 29.0 & 92.1 & 3.2 & 0.02 \\
\hline \multirow{3}{*}{$\begin{array}{l}\mathrm{H} 2 \text { (B) } \\
\quad+\text { post- } \\
\quad \text { optimization }\end{array}$} & 10 & 8.1 & 45.2 & 5.6 & 0.03 \\
\hline & 20 & 12.6 & 68.3 & 5.4 & 0.02 \\
\hline & 40 & 29.0 & 92.1 & 3.2 & 0.03 \\
\hline \multirow[t]{3}{*}{$\mathrm{H} 2(\mathrm{C})$} & 10 & 7.6 & 49.9 & 6.3 & 0.02 \\
\hline & 20 & 14.2 & 72.1 & 5.1 & 0.03 \\
\hline & 40 & 28.1 & 90.0 & 3.2 & 0.03 \\
\hline \multirow{3}{*}{$\begin{array}{l}\mathrm{H} 2(\mathrm{C}) \\
\quad+\text { post- } \\
\quad \text { optimization }\end{array}$} & 10 & 7.6 & 49.9 & 6.3 & 0.02 \\
\hline & 20 & 14.2 & 72.1 & 5.1 & 0.03 \\
\hline & 40 & 28.1 & 90.0 & 3.2 & 0.03 \\
\hline
\end{tabular}

words we never let the heuristics construct alignments for which this ratio would exceed $\lambda$. We successfully conducted experiments with $\lambda=2,4$ and 8 . We did not observe any noticeable difference in the algorithm performance between the various values of $\lambda$, except of course in the shape of the solution. For this reason we report in Table 1 computational results for $\lambda=2$. All statistics are average values over 10 randomly generated instances (i.e., different zone populations were generated). The table headings are as follows:

LMAX: maximal allowed length for the alignment;

Length: alignment length;

Ridership: total $O / D$ riderships covered by the alignment;

Ratio: ridership/length;

Seconds: computation time in seconds.

Two main conclusions can be drawn from Table 1. First the greedy alignment extension heuristic $H 1$ is much more effective than any of the greedy extension variants in producing a good quality solution. This is due to the fact that the solutions produced by $H 2$ tend to be $U$-shaped and very close to circular. After a few iterations this type of heuristic stops making substantial gains either in the alignment length or in the ridership. 
Another conclusion is that the use of a post-optimization phase after $\mathrm{H} 2$ does not yield any significant improvement. In other words, $H 1$ clearly dominates all variants of $H 2$. It is also worth observing that computation times are insignificant in all cases, which means that using $O / D$ information as opposed to catchment areas is computationally tractable. In practise, since one does not work with simulated data but with real information, estimating all $O / D$ demands should still result in a significant estimation and calibration exercise.

\subsection{Test on real context}

Sevillano (2003) recently applied the methodology described in this paper to determine the second most effective metro line in Sevilla in terms of trip coverage. Several scenarios were considered. We used two types of candidate-station distributions, two speed levels for surface traffic, four upper bound for distances between consecutive stations and three values of the logit parameter $\gamma$. This parameter was calibrated taking into account known values of the utilisation percentage of public transport in the city. Data were based on a mobility survey conducted in 2002. Two O/D matrices (for public and private transport modes) were obtained from previous data and 164 candidate nodes were selected.

Sevillano's conclusions are that H1, H2(A) and H2(A)+ (that is, H2(A) followed by a post-optimization step) are the best heuristics in this real-life context. The best algorithm depends on the upper bound imposed on inter-station spacing. Thus $\mathrm{H} 2(\mathrm{~A})+$ is best when this bound is small (750 and $1000 \mathrm{~m}$ ) and $\mathrm{H} 1$ is best when it is large (1500 $\mathrm{m})$. The two heuristics are equivalent for intermediate values $(1250 \mathrm{~m})$.

\section{Conclusions}

We have presented a model and some heuristics for the location of a rapid transit alignment where the objective is to maximize the total station-to-station ridership covered by the alignment. This objective is more realistic than the more traditional one based on catchment areas. It is also computationally feasible. Tests on randomly generated data have shown that a simple greedy extension heuristic yields the best results. Tests on the Sevilla data confirm this conclusion for scenarios where the upper bound on the interstation spacing is large. Otherwise an insertion heuristic followed by a post-optimization phase is best.

\section{Acknowledgments}

This research was in part supported by Canadian Natural Sciences and Engineering Research Council under grant OGP0039682 and by the Spanish Ministerio de Ciencia y Tecnología under grants BFM2000-1052-C02-01 and BFM2003-04062. Thanks are due to three referees and to Lisa Shepard for their valuable comments. 


\section{References}

Bouzaïene-Ayari, B., M. Gendreau, and S. Nguyen. (2001). "Modeling Bus Stops in Transit Networks: A Survey and New Formulations." Transportation Science 35, 304-321.

Brimberg, J. and R.F. Love. (1993). "A New Distance Function for Modeling Travel Distances on a Transportation Network." Transportation Science 27, 129-137.

Brimberg, J., R.F. Love, and J.H. Walker. (1995). "The Effect of Axis Rotation on Distance Estimation." European Journal of Operational Research 80, 357-364.

Bruno, G., M. Gendreau, and G. Laporte. (2002). "A Heuristic for the Location of a Rapid Transit Line." Computers \& Operations Research 29, 1-12.

Chapleau, R., P. Lavigueur, and K. Baass. (1986). "A Posteriori Impact Analysis of a Subway Extension in Montreal." Publication CRT-503, Centre de recherche sur les transports. Montreal, Canada.

Dufourd, H., M. Gendreau, and G. Laporte. (1996). "Locating a Transit Line Using Tabu Search.” Location Science 4, 1-19.

Garey, M.R. and D.S. Johnson. (1979). Computers and Intractability: A Guide to the Theory of NPCompleteness. Freeman, New York.

Gendreau, M., G. Laporte, and J.A. Mesa. (1995). "Locating Rapid Transit Lines." Journal of Advanced Transportation 29, 145-162.

Junta de Andalucía, Consejería de Obras Públicas y Transportes (1993). "Análisis de los corredores del área metropolitana de Sevilla y de los sistemas de transporte posibles." Private Report developed by SOFRETU Consulting and financed by Consejería de Obras Públicas y Transportes.

Laporte, G. (1995). “Comments on 'Multiobjective Routing Problems' by T.B. Boffey.” Top 3, 206-213.

Laporte, G., J.A. Mesa, and F.A. Ortega. (2000). "Optimization Methods for the Planning of Rapid Transit Systems." European Journal of Operational Research 122, 1-10.

Laporte, G., J.A. Mesa, and F.A. Ortega. (2002). "Locating Stations on Rapid Transit Lines." Computers \& Operations Research 29, 741-759.

Larson, R.C. and A.R. Odoni. (1981). Urban Operations Research. Prentice-Hall, Englewood Cliffs, New Jersey.

Love, R.F., J.G. Morris, and G.O. Wesolowsky. (1988). Facilities Location: Models and Methods. NorthHolland, New York.

Lutin, J.M. and G.P. Benz. (1992). "Key Issues in Light Rail Transit Station Planning and Design.” Transportation Research Records 1361, 117-124.

Magnanti, T.L. and R.T. Wong. (1984). "Network Design and Transportation Planning: Models and Algorithms." Transportation Science 18, 1-55.

Mesa, J.A. and F.A. Ortega. (2001). "Park-and-Ride Station Catchment Areas in Metropolitan Rapid Transit Systems." Mathematical Methods on Optimization in Transportation Systems. In M. Pursula and J. Niittymäki (eds.), Kluwer, Dordrecht, pp. 81-93.

Ortúzar, J.D. and L.F. Willumsen. (1990). Modelling Transport. Wiley, New York.

Sevillano, I. (2003). "Aplicación del concepto de cobertura de viaje al diseño y modelización de la segunda línea de metro de Sevilla" (in Spanish). Master's Dissertation, Engineers Higher Technical School, University of Seville, Spain.

Viegas, J.M. and A. Mexia. (1993). "Lisbon Metro Expands its Network." Public Transport International 4, 31-38.

Ward, J.E. and R.E. Wendell. (1980). "A New Form for Measuring Distances which Yields Linear Location Problems." Operations Research 28, 836-844.

Wirasinghe, S.C. and U. Vandebona. (1987). "Some Aspects of the Location of Subway Stations and Routes." Presented at the Fourth International Symposium on Locational Decisions (ISOLDE). Namur, Belgium. 